\title{
Patient controlled analgesia with remifentanil versus epidural analgesia in labour: randomised multicentre equivalence trial
}

\author{
(c) (1) (8) OPEN ACCESS
}

Liv M Freeman gynaecologist ${ }^{1}$, Kitty W Bloemenkamp gynaecologist ${ }^{1}$, Maureen T Franssen gynaecologist $^{2}$, Dimitri N Papatsonis gynaecologist ${ }^{3}$, Petra J Hajenius gynaecologist ${ }^{4}$, Markus W Hollmann professor of anaesthesiology ${ }^{5}$, Mallory D Woiski gynaecologist ${ }^{6}$, Martina Porath gynaecologist $^{7}$, Hans $\mathrm{J}$ van den Berg anaesthesiologist ${ }^{8}$, Erik van Beek gynaecologist ${ }^{9}$, Odette $\mathrm{W}$ $\mathrm{H}$ M Borchert anaesthesiologist ${ }^{10}$, Nico Schuitemaker gynaecologist ${ }^{11}$, J Marko Sikkema gynaecologist $^{12}$, A H M Kuipers anaesthesiologist ${ }^{13}$, Sabine L M Logtenberg midwife/PhD candidate ${ }^{14}$, Paulien C M van der Salm gynaecologist ${ }^{15}$, Katrien Oude Rengerink epidemiologist ${ }^{4}$, Enrico Lopriore neonatologist ${ }^{16}, \mathrm{M}$ Elske van den Akker-van Marle assistant professor of health economics ${ }^{17}$, Saskia le Cessie associate professor of medical statistics ${ }^{18}$, Jan M van Lith professor of obstetrics ${ }^{1}$, Michel M Struys professor of anaesthesiology ${ }^{19}$, Ben Willem $\mathrm{J} \mathrm{Mol} \mathrm{professor} \mathrm{of} \mathrm{obstetrics}{ }^{20}$, Albert Dahan professor of anaesthesiology ${ }^{21}$, Johanna M Middeldorp gynaecologist ${ }^{1}$

${ }^{1}$ Obstetrics, Leiden University Medical Centre, Leiden, Netherlands; ${ }^{2}$ Obstetrics and Gynaecology, University Medical Centre Groningen, Groningen, Netherlands; ${ }^{3}$ Obstetrics and Gynaecology, Amphia Hospital, Breda, Netherlands; ${ }^{4}$ Obstetrics and Gynaecology, Academic Medical Centre, Amsterdam, Netherlands; ${ }^{5}$ Anaesthesiology, Academic Medical Centre, Amsterdam, Netherlands; ${ }^{6}$ Obstetrics and Gynaecology, Radboud University Medical Centre, Nijmegen, Netherlands; ${ }^{7}$ Obstetrics and Gynaecology, Maxima Medical Centre, Veldhoven, Netherlands; ${ }^{8}$ Anaesthesiology, Maxima Medical Centre, Veldhoven, Netherlands; ${ }^{9}$ Obstetrics and Gynaecology, Saint Antonius Hospital, Nieuwegein, Netherlands; ${ }^{10}$ Anaesthesiology, Saint Antonius Hospital, Nieuwegein, Netherlands; ${ }^{11}$ Obstetrics and Gynaecology, Diakonessen Hospital, Utrecht, Netherlands; ${ }^{12}$ Obstetrics and Gynaecology, Hospital Group Twente, Almelo, Netherlands; ${ }^{13}$ Anaesthesiology, Hospital Group Twente, Almelo, Netherlands; ${ }^{14}$ Obstetrics and Gynaecology, Onze Lieve Vrouwe Gasthuis, Amsterdam, Netherlands; ${ }^{15}$ Obstetrics and Gynaecology, Meander Medical Centre, Amersfoort, Netherlands; ${ }^{16}$ Paediatrics, Leiden University Medical Centre, Leiden, Netherlands; ${ }^{17}$ Medical Decision Making, Leiden University Medical Centre, Leiden, Netherlands; ${ }^{18}$ Medical Statistics and Clinical Epidemiology, Leiden University Medical Centre, Leiden, Netherlands; ${ }^{19} \mathrm{Anaesthesiology,}$ University of Groningen and University Medical Centre Groningen, Groningen, Netherlands; ${ }^{20}$ Robinson Institute, School of Paediatrics and Reproductive Health, University of Adelaide, Australia; ${ }^{21}$ Anaesthesiology, Leiden University Medical Centre, Leiden, Netherlands

\author{
Abstract \\ Objective To determine women's satisfaction with pain relief using \\ patient controlled analgesia with remifentanil compared with epidural \\ analgesia during labour. \\ Design Multicentre randomised controlled equivalence trial. \\ Setting 15 hospitals in the Netherlands.
}

Participants Women with an intermediate to high obstetric risk with an intention to deliver vaginally. To exclude a clinically relevant difference in satisfaction with pain relief of more than $10 \%$, we needed to include 1136 women. Because of missing values for satisfaction this number was increased to 1400 before any analysis. We used multiple imputation to correct for missing data. 
Intervention Before the onset of active labour consenting women were randomised to a pain relief strategy with patient controlled remifentanil or epidural analgesia if they requested pain relief during labour.

Main outcome measures Primary outcome was satisfaction with pain relief, measured hourly on a visual analogue scale and expressed as area under the curve (AUC), thus providing a time weighted measure of total satisfaction with pain relief. A higher AUC represents higher satisfaction with pain relief. Secondary outcomes were pain intensity scores, mode of delivery, and maternal and neonatal outcomes. Analysis was done by intention to treat. The study was defined as an equivalence study for the primary outcome.

Results 1414 women were randomised, of whom 709 were allocated to patient controlled remifentanil and 705 to epidural analgesia. Baseline characteristics were comparable. Pain relief was ultimately used in $65 \%$ $(447 / 687)$ in the remifentanil group and 52\% (347/671) in the epidural analgesia group (relative risk $1.32,95 \%$ confidence interval 1.18 to 1.48 ). Cross over occurred in 7\% (45/687) and $8 \%(51 / 671)$ of women, respectively. Of women primarily treated with remifentanil, $13 \%(53 / 402)$ converted to epidural analgesia, while in women primarily treated with epidural analgesia 1\% (3/296) converted to remifentanil. The area under the curve for total satisfaction with pain relief was 30.9 in the remifentanil group versus 33.7 in the epidural analgesia group (mean difference -2.8, $95 \%$ confidence interval -6.9 to 1.3$)$. For who actually received pain relief the area under the curve for satisfaction with pain relief after the start of pain relief was 25.6 in the remifentanil group versus 36.1 in the epidural analgesia group (mean difference $-10.4,-13.9$ to -7.0 ). The rate of caesarean section was $15 \%$ in both groups. Oxygen saturation was significantly lower $\left(\mathrm{SpO}_{2}<92 \%\right)$ in women who used remifentanil (relative risk 1.5, 1.4 to 1.7). Maternal and neonatal outcomes were comparable between both groups.

Conclusion In women in labour, patient controlled analgesia with remifentanil is not equivalent to epidural analgesia with respect to scores on satisfaction with pain relief. Satisfaction with pain relief was significantly higher in women who were allocated to and received epidural analgesia.

Trial registration Netherlands Trial Register NTR2551.

\section{Introduction}

Epidural analgesia is considered to be the most effective method of pain relief during labour and is often the preferred choice of analgesia. ${ }^{1}$ Intramuscular or intravenous opioids can provide an alternative in situations where regional analgesia is unavailable or contraindicated or if less invasive methods are preferred by the woman or obstetrician. ${ }^{2}$ Remifentanil is a potent $\mu$ opioid receptor agonist. Its short context sensitive half life (3-4 minutes) and short elimination half time (10-20 minutes) make it suitable for administration under the control of the patient for women in labour who want pain relief. ${ }^{3}$ Placental transfer of remifentanil occurs, but the opioid is rapidly metabolised and redistributed by the fetus. ${ }^{4}$

Although epidural analgesia during labour is the preferred method because it provides superior analgesia to systemic opioids, various studies show comparable maternal satisfaction with patient controlled remifentanil. ${ }^{56}$ Two previous studies that assessed satisfaction with pain relief with patient controlled remifentanil compared with epidural analgesia reported no differences. Both studies, however, had limitations. Volmanen and colleagues limited the observation period to only one hour after the start of pain relief, ${ }^{5}$ while Douma and colleagues recorded pain relief scores as a secondary outcome in a study powered to investigate difference in pain scores. ${ }^{6}$ In both studies, epidural analgesia was superior to patient controlled remifentanil in terms of pain intensity.
The most recent Cochrane review on this topic recommended a randomised controlled trial to examine patient controlled analgesia with an opioid compared with other methods of analgesia and to report on maternal satisfaction, cointerventions, and maternal and neonatal outcomes. ${ }^{7}$ We conducted this study to test the hypothesis that patient controlled remifentanil is equivalent to epidural analgesia with respect to satisfaction with pain relief.

\section{Methods \\ Design}

We performed a multicentre randomised clinical trial within the Dutch consortium for women's health and reproductivity (NTR 2551). The study was performed in three academic hospitals, 11 teaching hospitals, and one general hospital. In the Netherlands healthy low risk pregnant women start antenatal care in primary midwifery led care. When medical complications occur, either maternal or fetal, women are referred to secondary or tertiary care. For this study we recruited only women in secondary and tertiary care (intermediate/high risk). Women are considered low risk if their medical and/or obstetric history is uneventful. Women are considered intermediate or high risk if they have illnesses in their medical history that can affect pregnancy or that are affected by pregnancy or if they have complications in this or previous pregnancies or deliveries.

Women were eligible to participate if they were healthy or had a mild systemic disease (American Society of Anesthesiologists physical classification 1 or 2$),{ }^{8}$ were aged 18 or older, and were scheduled to deliver vaginally after 32 weeks. Exclusion criteria were contraindications for epidural analgesia or hypersensitivity to one of the drugs used.

After informed consent, but before the onset of active labour, women were randomly allocated to patient controlled remifentanil or epidural analgesia. All women were randomised before the start of actual labour. As analgesia during labour was given only if it was requested, not all women received pain relief.

\section{Interventions}

\section{Remifentanil group}

The patient controlled device was programmed to deliver $30 \mu \mathrm{g}$ remifentanil (solution $20 \mu \mathrm{g} / \mathrm{mL}$ ) on request with a lockout time of three minutes. This dose regimen was based on previous studies. ${ }^{69}$ The dose could be increased to $40 \mu \mathrm{g}$ in case of insufficient pain relief or decreased to $20 \mu \mathrm{g}$ in case of excessive side effects. No background infusion was allowed. Women who were treated patient controlled remifentanil were instructed on how to use the device and to maximise analgesia by pressing the device's button in anticipation of the next contraction. Remifentanil has a rapid onset of action and short context sensitive half life, thus administration of a bolus dose in anticipation of the next contraction ensures maximum effect. ${ }^{3}$ If pain relief was inadequate, women could request epidural analgesia. They were advised to discontinue using the device during the second stage of labour to minimise the risk of neonatal side effects.

\section{Epidural analgesia group}

Women randomised to epidural analgesia received this when they requested pain relief, according to local protocol. If pain relief after epidural analgesia was judged inadequate by the woman, she could receive patient controlled remifentanil instead 
of epidural analgesia. No advice was given regarding continuing epidural analgesia during the second stage of labour. Dutch guidelines advise the continuation of epidural analgesia during second stage provided there is no effect on motor function. ${ }^{1}$

\section{Data collection}

During labour, women were asked two separate questions. They were asked to rate their satisfaction with pain on a specially designed ruler with a visual analogue scale ranging from 0 (highly dissatisfied) to $100 \mathrm{~mm}$ (highly satisfied). They started from the beginning of actual labour and were asked to report hourly. In addition, they were asked to rate the pain intensity score during contractions every hour on a scale ranging from 0 (no pain) to $100 \mathrm{~mm}$ (worst pain imaginable).

For satisfaction with pain relief, women were asked to rate their satisfaction with pain (relief) ("how would you rate your satisfaction with pain relief?") on a visual analogue scale. This was described briefly in the patient information given to the women before randomisation and in detail at admission for delivery. For the pain intensity score, women asked to rate their pain score ("how would you rate your pain during a contraction?") on a different visual analogue scale.

Written examples of these questions and how to use the VAS ruler were available at the labour ward (see appendix 1). After birth, women were asked to rate their overall satisfaction with the pain during labour on an 11 point numerical rating scale as a measure of the overall pain experience. They were not asked to rate the overall experience of labour.

Maternal oxygen saturation was monitored continuously in women receiving pain relief. The following measurements were obtained and recorded once before the start of analgesia and at 15 minute intervals during the first hour of treatment followed by hourly recordings until delivery: maternal temperature, blood pressure, heart rate and respiratory rate, and oxygen saturation determined by pulse oximetry.

In women who received analgesia, the nurse, midwife, or obstetrician recorded the incidence of nausea, vomiting, itching, hypotension (systolic blood pressure $<90 \mathrm{~mm} \mathrm{Hg}$ ), desaturation $\left(\mathrm{SpO}_{2}<92 \%\right)$, and respiratory depression (frequency $<8 / \mathrm{min}$ ). Additional measures were advised in case of hypotension, maternal desaturation, or respiratory depression.

Fetal heart rate and uterine activity were measured with external fetal cardiotocography or fetal scalp electrode and intrauterine pressure device.

\section{Outcomes}

The primary outcome measure was satisfaction with pain relief measured on a visual analogue scale ranging from 0-100 mm. Satisfaction was expressed as the area under the pain satisfaction curve, which is a summary measure that integrates serial assessments of a woman's satisfaction with pain relief over the duration of the study. The area under the curve (AUC) is a measure that is often used in clinical pharmacology, but it can also be used for clinical endpoints-for example, the use in pain assessment. ${ }^{10-13}$ A higher AUC represents a higher satisfaction with pain relief. The AUC was calculated for the duration of labour and for the time that pain relief was administered. The AUC could be calculated if a woman had rated satisfaction with pain relief on at least two different time points.

Our published protocol stated that both effectiveness and cost effectiveness were primary outcome measures. Satisfaction with pain relief was the primary outcome measure for effectiveness from the start of the study. We planned to perform a cost effectiveness analysis as well, taking into account the primary outcome for effectiveness. Because this was not made clear enough in the original protocol and registry it was changed in the last amended protocol. This last amended protocol was submitted before the last randomised woman delivered and as a result we did not have access to the data. ${ }^{14}$

Secondary outcome measures were the AUC for pain intensity scores, score for overall satisfaction with pain relief during labour, the highest pain intensity score during labour, pain intensity and satisfaction with pain relief at the moment of request for pain relief, highest score for satisfaction with pain relief after pain relief was used, and the mean scores of pain and satisfaction with pain relief.

We also recorded characteristics of labour (time from request to start of analgesia, duration of analgesia, duration of second stage, use of oxytocin, mode of delivery, reasons for instrumental delivery), maternal outcomes (postpartum haemorrhage (estimated blood loss $>1000 \mathrm{~mL}$ ), suspicion of intrapartum infection (temperature $>38.0^{\circ} \mathrm{C}$ and the use of antibiotics), spinal headache, major maternal complications, maternal parameters (temperature, blood pressure, oxygen saturation, and respiratory rate)), and maternal admission. For the neonate we assessed Apgar score at 5 minutes, arterial cord blood $\mathrm{pH}$, neonatal admission, and reasons for neonatal admission.

\section{Sample size calculation}

We calculated our sample size based on the primary outcome measure of satisfaction with pain relief, assuming that there would be no difference in satisfaction (two sided test, $\alpha 0.05$, power 0.9 ). In this equivalence design, we would need 102 women to be treated in each group to exclude a potential clinically relevant difference of $10 \%$ (on an 11 point scale, estimated SD 2.2). Allowing for $30 \%$ and $10 \%$ cross over in the remifentanil group and epidural analgesia group, respectively, we needed 568 women in total. We estimated that out of pregnant women who would be willing to participate in the study, about half would actually request analgesia. We therefore needed to randomise 1136 women. In anticipation of missing data on the primary endpoint during the study period, we extended the number of women to 1400 before any comparative analysis.

This sample size was calculated based on a visual analogue scale for satisfaction with pain relief at one time point. In February 2013 we amended the protocol because we decided to change the primary outcome from a score at one time point to the area under the satisfaction curve, which integrates all visual analogue scores measured over time. In our opinion the AUC best represents the overall satisfaction with pain relief and it can deal with missing values, but at the start of the trial we were not well enough aware of this.

For this measure we also judged that $10 \%$ equals a clinically relevant difference. Our sample size calculation was done on a different outcome parameter. In the sample size calculations, we used $10 \%$ reduction on the visual analogue scale for satisfaction with pain relief as equivalence margin, which is one point reduction in satisfaction with pain relief. As we changed the primary outcome measure to a time weighted measure by using the AUC, we could no longer use this equivalence margin. We therefore used an equivalence margin of $10 \%$ of the mean AUC. 


\section{Interim analysis and stopping rules}

We used specially designed forms to report serious adverse events and suspected unexpected serious adverse reactions to the ethics committee of the Leiden University Medical Centre. A data safety monitoring board was established before the start of the trial. No interim analysis was performed because of the equivalence design of the trial. Serious adverse events and reactions were reported to the board and medical ethics committee to evaluate the safety of women. Predefined serious adverse events were requirement for mechanical ventilation or cardiopulmonary resuscitation, meningitis, and epidural haematoma. Apart from that we asked to be informed about respiratory depression $<8$ breaths/minute and oxygen saturation $<92 \%$ that did not respond to a decrease in bolus dose. These events had to be reported to the principal investigator and to the data safety monitoring board. If deemed necessary the data safety monitoring board would be able to (temporarily) stop the study.

\section{Randomisation and blinding}

Randomisation was performed through a web based randomisation program. We randomised in fixed blocks of three, stratified for centre and parity. The allocation code appeared after a patient's initials were entered into the randomisation program.

All women were randomised before the start of labour. If women requested pain relief during labour, the allocated intervention was provided. Before labour, women did not have the option of choosing analgesia other than according to randomisation. Blinding was not possible because of the nature of the two interventions. Research nurses/midwives as well as attending medical staff performed randomisation.

\section{Data analysis}

The trial was designed as an equivalence trial for the primary outcome measure AUC of satisfaction with pain relief and the secondary outcome measure AUC of pain intensity. The other secondary outcomes were analysed for superiority. Our null hypothesis was that the difference in the score for satisfaction with pain relief, scored on a visual analogue scale (0-100 mm), between the two study groups would be greater than $10 \%$. Preliminary unpublished data in perioperative patients using patient controlled opioid treatment had shown that changes (that is, increases) in pain intensity scores of $10 \%$ or larger will prompt action in a patient - that is, he or she will require additional pain relief by pressing the device's button.

Extrapolation of these data to the current study suggests that at differences in visual analogue scores of $10 \%$ or more, clinical differences in satisfaction with pain relief can be assumed. We calculated the estimated standard deviation using data from Volmanen and colleagues ${ }^{5}$ and converted those from a five point to an 11 point scale.

Data were analysed on an intention to treat basis. We tested for equivalence by determining whether the upper and lower limits of the two sided $95 \%$ confidence interval on the primary endpoint AUC of satisfaction with pain relief and the secondary endpoint AUC of pain intensity did not exceed the equivalence margin of $10 \%$. Normally distributed data were presented as means with SDs; skewed distributions were presented as medians with interquartile range. For categorical data, the treatment effect was presented as relative risk with $95 \%$ confidence intervals. For secondary outcome measures we calculated $\mathrm{P}$ values with the $\chi^{2}$ test, unless the expected cell count was less than 5, in which case we used the Fisher's exact test. For continuous data with a non-normal distribution, we used the Mann-Whitney U test.

Calculation of the percentages was based on the number of valid observations. Statistical analysis was performed with SPSS version 20 (SPSS, Chicago, IL). P<0.05 was considered significant.

We performed two analyses. Firstly, we analysed the whole group of randomised women on an intention to treat basis. This analysis included women who did and did not receive any pain relief (687 in remifentanil group, 671 in epidural group). In a second analysis, we included only those women who actually received pain relief (447 in remifentanil group, 347 in epidural group). To correct for possible confounding in the second analysis because a larger number of women who received pain relief were in the group allocated to remifentanil, we also compared the two randomisation groups in the subgroup of women who actually received pain relief using multiple linear regression, with adjustment for randomisation outcome, age, race, education, parity, onset of labour, dilation at request of pain relief, and premature labour.

\section{Subgroup analyses}

We planned subgroup analyses for satisfaction with pain relief for nulliparous women versus multiparous women, previous caesarean section, spontaneous versus induced labour, educational level, aged under 36 versus 36 or older, gestational age at delivery ( $<34$ weeks, $34-37$ weeks, $>37$ weeks), and singleton versus multiple pregnancy.

\section{Missing data}

We used multiple imputation with SPSS to correct for missing primary outcome data. ${ }^{15-17} \mathrm{We}$ imputed missing AUC values for satisfaction with pain relief and pain intensity (transformed so that the distribution was approximately normal) using 20 imputed datasets. Other missing values were not imputed.

\section{Results}

Between 30 May 2011 and 24 October 2012, we randomised 1414 women to receive patient controlled remifentanil $(n=709)$ or epidural analgesia $(n=705)$ should they request analgesia during labour. After randomisation, we excluded 51 women (22 in remifentanil group, 29 in epidural group) because of elective caesarean section.

In the epidural group, three women were lost to follow-up, while two withdrew informed consent after randomisation. We analysed the data from 1358 women, 687 in the remifentanil group and 671 in the epidural group (figure $\Downarrow$ ). Baseline characteristics were comparable between groups (table $1 \Downarrow$ ).

Of the 709 women randomised to patient controlled remifentanil, $447(65 \%)$ actually received analgesia during labour, compared with $52 \%$ (347) in the epidural analgesia group (relative risk $1.32,95 \%$ confidence interval 1.18 to 1.48 ).

Of the 447 women in the remifentanil group who received pain relief, 402 women received immediate remifentanil. Forty five women received analgesia other than the allocated type: 41 received epidural analgesia, and four received other opioids. Of the 402 women who started remifentanil, 53 women converted to epidural analgesia because of insufficient analgesia (figure $\Downarrow$ ). Of women who were treated with remifentanil, 92\% (411/447) started with a dose of $30 \mu \mathrm{g}$; the other women were given an initial dose of 20-40 $\mu$ gr. In $13 \%$ (59/447) the dose was increased once, and in 3\% (14/447) it was decreased once. In 3\% (12/447) 
and $0.7 \%(3 / 447)$ the bolus dose was increased twice or three times, respectively.

Of the 347 women who requested pain relief and been allocated to epidural analgesia, 296 received immediate epidural analgesia. Fifty one women were received analgesia other than allocated type: 33 were treated with remifentanil (of whom two women converted back to epidural analgesia after remifentanil), and 18 received other opioids. Three women initially treated with epidural analgesia converted to remifentanil because of insufficient analgesia (figure $\Downarrow$ ).

The epidural regimens used were ropivacaine/sufentanil (37\%), bupivacaine/sufentanil (46\%), levobupivacaine/sufentanil (6\%), and bupivacaine/fentanyl (11\%).

Reasons for non-compliance (treatment other than the randomised outcome) included doctors' or patients' preference/request, expectation of quick or slow delivery, logistical problems (for instance, non-availability of the anaesthetist (within one hour)), and new contraindication for randomised treatment (table $2 \Downarrow$ ).

\section{Missing data}

We could calculate the primary outcome measure, AUC for satisfaction with pain relief during active labour, for $57 \%$ of women in the remifentanil group and $43 \%$ in the epidural group. In the subgroup of women who received analgesia, the AUC for satisfaction with pain relief during administration of pain relief could be calculated for $71 \%$ and $57 \%$, respectively.

The AUC for pain intensity score during active labour could be calculated for $64 \%$ of participants in the remifentanil group and $53 \%$ in the epidural group. In the subgroup of women who received analgesia the AUC for pain intensity score could be calculated for $77 \%$ of women in the remifentanil group and $63 \%$ in the epidural group. Characteristics of complete and incomplete cases are in appendix 2.

\section{Primary outcome}

The AUC for satisfaction with pain relief during labour for all randomised women was lower in the remifentanil group (difference $-2.8,95 \%$ confidence interval -6.9 to 1.3 ). As this does not exclude a potential clinically relevant difference, we cannot conclude that the treatments are equivalent. Furthermore, in the subgroup of women who actually received analgesia, the AUC for satisfaction with pain relief after start of pain relief was significantly lower in women who asked for pain relief and were randomised to remifentanil (difference $-10.4,-13.9$ to -7.0) (table $3 \Downarrow$ ).

The AUC for pain intensity during labour for all randomised women was higher in the remifentanil group (difference 3.8, $95 \%$ confidence interval 0.92 to 6.6). For the group of women who actually received analgesia, the AUC for pain intensity after the start of pain relief was significantly higher in women who requested pain relief and were randomised to remifentanil (difference $6.4,3.5$ to 9.4 ) (table $3 \Downarrow$ ).

Table $3 \Downarrow$ also shows the values without imputation for the AUC, providing a larger effect size than the imputed values. Results of the comparisons in the group of women who actually received analgesia, with adjustment for possible confounders, were similar: the difference in AUC for satisfaction with pain relief after the start pain relief was -8.7 (95\% confidence interval -12.0 to -5.5$)$ and the difference in AUC for pain score after the start pain relief was 7.6 (4.8 to 10.4$)$.

\section{Secondary outcomes Overall scores and means}

The overall satisfaction score with pain during labour was not significantly different between the study groups in the intention to treat analysis, when we accounted for scores of women with and without pain relief (6.9 remifentanil $v 7.2$ epidural, difference $-0.29,95 \%$ confidence interval -0.60 to 0.01 ). In women who received pain relief the overall satisfaction score was significantly lower for women randomised to remifentanil: 6.8 for women randomised to remifentanil $v 7.3$ for women randomised to epidural analgesia (difference $-0.52,95 \%$ confidence interval -0.91 to -0.13 ).

Mean scores for satisfaction with pain relief were significantly lower in the remifentanil group, both for the total period of active labour and after the start of pain relief. Mean pain scores for both periods were significantly higher in the remifentanil group. Pain scores and satisfaction with pain relief at the time when pain relief was requested were not significantly different between the groups. Highest satisfaction with pain relief and lowest pain intensity score after pain relief were significantly different in favour of epidural analgesia (table $4 \Downarrow$ ). These scores were not imputed.

\section{Characteristics of labour and maternal and neonatal outcomes}

The intervals from request for pain relief to the start of pain relief and from start of pain relief to delivery were shorter in the remifentanil group (table $5 \Downarrow$ ). There were no other significant differences in characteristics of labour and maternal and neonatal outcomes between the two study groups (table $5 \Downarrow$ ).

In women who actually received analgesia, the only significant difference in characteristics of labour and maternal and neonatal outcomes was a shorter duration of second stage of labour in women randomised to remifentanil (median duration 25, interquartile range 11-51, minutes) compared with epidural analgesia $(34,15-60 ; \mathrm{P}=0.01)$.

Some side effects were reported more often in women who received analgesia. Temperature was significantly higher and hypotension more common in the women who received epidural analgesia. Oxygen saturation was significantly lower with remifentanil. There were four reported cases of respiratory depressions of $<8$ breaths a minute in the remifentanil group and none in the epidural group. Nausea was more common in the group randomised to remifentanil, but vomiting and itching were not (table $6 \Downarrow$ ).

One serious adverse event was recorded: one woman who received epidural analgesia presented with eclampsia on the fourth day after delivery. There were no maternal deaths. Postpartum admission, duration of admission, and reasons for admission for mothers and neonates were comparable in both groups (table $5 \Downarrow$ ).

There were three intrauterine fetal deaths after randomisation, all before the start of labour. These were two singletons at a gestational age 41-42 weeks and the second twin of monochorionic twins at $35+6$ with suspicion of acute twin to twin transfusion syndrome. Three neonates died postpartum, two singletons and one twin, all from congenital defects that were diagnosed during pregnancy (Zellweger syndrome, two major cardiac defects).

Subgroup analyses for AUC for pain and satisfaction with pain relief were performed as planned for all predescribed groups except for gestational age at birth 32-34 weeks because only one woman in the remifentanil group received pain relief. 
Results of subgroup analysis were similar to those of the whole group, with no significant interactions found (see figs A-D in appendix 3).

\section{Discussion}

\section{Statement of principal findings}

The results of this large multicentre trial show that patient controlled analgesia with remifentanil is not equivalent to epidural analgesia with respect to satisfaction with pain relief, with poorer scores obtained in women treated with remifentanil. This study also confirms the results of previous trials that epidural analgesia provides superior pain relief when measured in terms of pain intensity scores. ${ }^{5618-20}$ These results were consistent throughout all subgroups. In contrast with previous studies that did not have sufficient power to detect a difference, this is the first well powered study to showing that patient controlled remifentanil is not equivalent to epidural analgesia with respect to satisfaction with pain relief.

Significantly more women randomised to remifentanil actually requested and received analgesia. We relate this to the perception of women that remifentanil is less invasive and more easily available. Furthermore, the time between the request for and start of analgesia was shorter in the remifentanil group, probably because an anaesthetist is not required.

Duration of analgesia (that is, the time from start of analgesia to birth) was significantly longer in the epidural analgesia group. One explanation might be the epidural analgesia slows labour but there are other possible explanations. For example, in the Netherlands it is still practice to wait for the urge to push (and even to stop the epidural to increase sensation). This might cause a delay in starting the second stage of labour.

An important finding from the secondary outcome measures is the high incidence of desaturations, with oxygen saturations below $92 \%$ in $18 \%$ and below $95 \%$ in $38 \%$ of women treated with remifentanil, compared with $5 \%$ and $12 \%$ in women treated with epidural analgesia (table $6 \Downarrow$ ).

There were four reported respiratory depressions with $<8$ breaths a minute in the remifentanil group, all during administration of remifentanil. Although the difference in occurrence is not significant, probably because of the low prevalence of respiratory depression, and although there were about $25 \%$ missing values, this is a potentially life threatening side effect of remifentanil. Caregivers should be aware that serious respiratory complications can occur during administration of remifentanil (table $6 \Downarrow)^{19-22}$

\section{Strengths and weaknesses}

In the Netherlands there is a distinction between primary and secondary/tertiary care in obstetrics. Women at low risk are under antenatal care of community midwives; intermediate or high risk women are under antenatal care of gynaecologists. We included only women in secondary/tertiary care as we assumed that opinions on labour and pain (satisfaction) are different not only in the women but also in the obstetric team. As we were interested in this possible difference, we started a second study in low risk women in primary care. This study has been completed and is under analysis.

We decided to use the area under the curve (AUC) as our primary outcome as it included all available data from responders and can be interpreted as an integral measure of total satisfaction with pain relief rather than using satisfaction only at a specific time point. The AUC gives a time weighted measure of total satisfaction with pain relief.
We measured satisfaction scores at one hour intervals during active labour and used the AUC as a time weighted measure of this index. Use of AUC requires multiple scores during labour. This could have resulted in an increase in missing data as in some women, especially those women who did not receive pain relief, often just one measurement was available. Still we chose this approach as pain AUC gives a time weighted and consequently a more reliable measure of pain response than single measurements.

Though we believe that a time weighted measure is the best way to measure total satisfaction with pain relief, pain relief was administered over a longer period of time in the epidural analgesia group. This influences the AUC but it also influences total pain experience (with a higher total satisfaction over a longer period of time). To test if the difference in AUC between the two study groups during administration of pain relief was influenced by this difference we also analysed the AUC per hour and mean satisfaction with pain relief on specific time points. As these were also significantly lower in the remifentanil group, we believe the total AUC is only minimally influenced by this extra time.

The main weakness of our study was the percentage of missing values for satisfaction with pain relief and pain intensity. The AUC for satisfaction with pain relief during active labour could be calculated for $57 \%$ of women in the remifentanil group and $43 \%$ in the epidural group. In the subgroup of women who actually received analgesia, the AUC for satisfaction with pain relief during administration of pain relief could be calculated for $71 \%$ and $57 \%$, respectively.

As mentioned above, multiple measurements are necessary to calculate the AUC. One explanation for the missing data is reluctance of caregivers to focus on pain in women who are not asking for pain relief. Another reason might be that epidural analgesia is routine so extra measurements are more easily forgotten. We opted to use imputation to correct for these missing values, assuming that scores for satisfaction with pain relief were missing at random. Hence, we judged that imputation would give a more accurate representation of total satisfaction with pain relief than the exclusion of women with just one or no data points. ${ }^{15}$ The groups with complete and incomplete data were similar on all baseline characteristics and most labour characteristics. They were, however, significantly different on onset of labour, request for pain relief, and mode of delivery, with fewer scores obtained from women in spontaneous labour who did not receive pain relief and delivered spontaneously (appendix 2). This could be explained by shorter duration of labour and shorter time in hospital for those women.

Furthermore, analyses without imputed values showed similar differences in AUC for pain intensity and satisfaction with pain relief.

As randomisation was performed antenatally, women knew their allocated intervention when in need for pain relief during labour. We chose this design to mimic daily practice, where a woman knows which methods of pain relief are available and which one she will most likely receive. Because masking of treatment was considered unethical, crossovers might have occurred because of preferences of doctors or women for one of the two treatments in light of labour characteristics. This could have influenced study outcome to some extent. But as the percentage of non-compliance in both groups was around $10 \%$, lower than the number we anticipated in the power analysis, we think this influence was minimal. Furthermore, in an equivalence design non-compliance will provide an underestimation of the effect, making it more plausible that there truly is no equivalence between both interventions. 


\section{Explanation and implication for clinicians and policymakers}

Although patient controlled remifentanil does improve pain and scores for satisfaction with pain relief, our study shows that this improvement is not optimal when compared with improvement of scores with epidural analgesia. As scores for satisfaction with pain relief were lower and pain intensity scores higher in women randomised to remifentanil, we cannot suggest it as an equivalent alternative to epidural analgesia. The higher percentage of women who actually received pain relief in the remifentanil group could suggest that there is a need for other types of analgesia options besides epidural analgesia and that women and/or caregivers perceive remifentanil as less invasive and hence easier to administer and possibly also less harmful. Another explanation might be that remifentanil is more readily available than epidural analgesia because the presence of an anaesthetist is not required. Either patient controlled remifentanil is a much needed addition to the possibilities of analgesia or we should still make epidural analgesia more accessible for all women who request pain relief during labour.

Delivery outcome and labour characteristics were not different between groups nor were maternal and neonatal morbidity. But we did find a significant difference in respiratory side effects in women treated with remifentanil. Remifentanil is a potent opioid and should be used with appropriate monitoring and the ability to intervene if respiratory complications arise. ${ }^{20}{ }^{21}$ Women should be counselled on the effects and side effects of both remifentanil and epidural analgesia.

Contributors: All authors have made substantial contributions to the conception or design of the work or the acquisition, analysis, or interpretation of the data. They were actively involved in drafting the work or critically revising it. They approved the final version of the manuscript for publication and agree to be accountable for all aspects of the work. LMF is guarantor.

Funding: This study was funded by a grant from ZonMW (Dutch Organization for Health Care Research and Development) project No 80-82310-97-11039.

Competing interests: All authors have completed the ICMJE uniform disclosure form at www.icmje.org/coi_disclosure.pdf and declare: no support from any organisation for the submitted work; no financial relationships with any organisations that might have an interest in the submitted work in the previous three years; no other relationships or activities that could appear to have influenced the submitted work.

Ethical approval: This study was approved by the central committee on research involving human subjects and the medical ethics committee of Leiden University Medical Centre (p10-240) as well as the medical ethics committees of all participating hospitals and written informed consent was given by all women at antenatal visits before the onset of labour.

Data sharing: Relevant patient level data are available from the corresponding author on reasonable request. Informed consent for data sharing was not obtained but the presented data are anonymised.
Transparency declaration: LMF affirms that this manuscript is an honest, accurate, and transparent account of the study being reported; that no important aspects of the study have been omitted; and that any discrepancies from the study as planned have been explained.

$1 \quad$ NVOG (Dutch Society of Obstetrics and Gynaecology). Guideline Pijnstilling tijdens de bevalling [pain relief during labour]. NVOG, 2008.

2 Douma MR, Verwey RA, Kam-Endtz CE, van der Linden PD, Stienstra R. Obstetric analgesia: a comparison of patient-controlled meperidine, remifentanil, and fentanyl in labor. Br J Anaesth 2010;104:209-15.

3 Michelsen LG, Hug CC Jr. The pharmacokinetics of remifentanil. J Clin Anesth 1996;8:679-82

4 Kan RE, Hughes SC, Rosen MA, Kessin C, Preston PG, Lobo EP. Intravenous remifentanil. Anaesthesiology 1998;88:1467-749

5 Volmanen P, Sarvela J, Akural EI, Raudaskoski T, Korttila K, Alahuhta S. Intravenous remifentanil vs. epidural levobupivacaine with fentanyl for pain relief in early labour: a randomised, controlled, double-blinded study. Acta Anaesthesiol Scand 2008:52:249-55

6 Douma MR, Middeldorp JM, Verwey RA, Dahan A, Stienstra R. A randomised comparison of intravenous remifentanil patient-controlled analgesia with epidural ropivacaine/sufentanil during labour. Int J Obstet Anesth 2011;20:118-23.

7 Ullman R, Smith LA, Burns E, Mori R, Dowswell T. Parenteral opioids for maternal pain management in labour. Cochrane Database Syst Rev 2011:10:CD007396.

8 American Society of Anesthiologists. ASA physical status classification system. ASA, 2015. www.asahq.org/resources/clinical-information/asa-physical-status-classificationsystem.

9 Blair JM, Dobson GT, Hill DA, McCracken GR, Fee JPH. Patient controlled analgesia for labour: a comparison of remifentanil with pethidine. Anesthesia 2005;60:22-7.

10 On Biostatistics and Clinical Trials. Using are under the curve (AUC) as clinical endpoints. 2014. http://onbiostatistics.blogspot.nl/2012/10/using-area-under-curve-auc-as-clinical. html.

11 Cappelleri JC, Bushmakin AG, Zlateva G, Sadosky A. Pain responder analysis: use of area under the curve to enhance interpretation of clinical trial results. Pain Practice 2009;9:348-53.

12 Barden J, Edwards J, Moore RA, McQuay HJ. Single dose oral diclofenac for postoperative pain. Cochrane Database Syst Rev 2004;2:CD004768.

13 Toms L, McQuay HJ, Derry S Moore RA. Single dose oral paracetamol (acetaminophen) for postoperative pain in adults. Cochrane Database Syst Rev 2008;4:CD004602.

14 Freeman LM, Bloemenkamp KW, Franssen MT, Papatsonis DN, Hajenius PJ, van Huizen $\mathrm{ME}$, et al. Remifentanil patient controlled analgesia versus epidural analgesia in labour. A multicentre randomized controlled trial. BMC Pregnancy Childbirth 2012;12:63.

15 Donders AR, van der Heijden GJ, Stijnen T, Moons KG. Review: a gentle introduction to imputation of missing values. J Clin Epidemiol 2006;59:1087-91

16 Van der Heijden GJ, Donders AR, Stijnen T, Moons KG. Imputation of missing values is superior to complete case analysis and the missing-indicator method in multivariable diagnostic research: a clinical example. J Clin Epidemiol 2006;59:1102-9.

17 Greenland S, Finkle WD. A critical look at methods for handling missing covariates in epidemiologic regression analyses. Am J Epidemiol 1995;142:1255-64.

18 Liu ZQ, Chen XB, Li HB, Qiu MT, Duan T. A comparison of remifentanil parturient-controlled intravenous analgesia with epidural analgesia: a meta-analysis of randomized controlled trials. Anesth Analg 2014;118:598-603.

19 Schnabel A, Hahn N, Broscheit J, Muellenbach RM, Rieger L, Roewer N, et al. Remifentanil for labour analgesia: a meta-analysis of randomised controlled trials. Eur $J$ Anaesthesio 2012;29:177-85.

20 Stocki D, Matot I, Einav S, Eventov-Friedman S, Ginosar Y, Weiniger CF. A randomized controlled trial of the efficacy and respiratory effects of patient-controlled intravenous remifentanil analgesia and patient-controlled epidural analgesia in laboring women. Anesth Analg 2014;118:589-97.

21 Marr R, Hyams J, Bythell V. Cardiac arrest in an obstetric patient using remifentani patient-controlled analgesia. Anaesthesia 2013:68:283-7.

22 Kranke P, Girard T, Lavand'homme P, Melber A, Jokinen J, Muellenbach RM, et al. Must we press on until a young mother dies? Remifentanil patient controlled analgesia in labour may not be suited as a "poor man's epidural". BMC Pregnancy Childbirth 2013;13:139.

Accepted: 16 January 2015

\section{Cite this as: BMJ 2015:350:h846}

This is an Open Access article distributed in accordance with the Creative Commons Attribution Non Commercial (CC BY-NC 4.0) license, which permits others to distribute, remix, adapt, build upon this work non-commercially, and license their derivative works on different terms, provided the original work is properly cited and the use is non-commercial. See: http://creativecommons.org/licenses/by-nc/4.0/. 


\section{What is already known on this topic}

Epidural analgesia is considered to be the most effective method of pain relief during labour Satisfaction with pain relief might be comparable between patient controlled remifentanil and epidural analgesia

\section{What this study adds}

Patient controlled remifentanil is not equivalent to epidural analgesia and performs worse in terms of women's satisfaction with pain relief

Significantly more women randomised to remifentanil actually requested and received analgesia

As more women allocated to remifentanil had respiratory complications, caregivers should be aware that such complications can occur with this drug

\section{Tables}

Table 1| Baseline characteristics of pregnant women allocated to patient controlled remifentanil or epidural analgesia in labour. Figures are numbers (percentage) unless otherwise indicated

\begin{tabular}{lcc} 
& Remifentanil $(\mathbf{n}=\mathbf{6 8 7})$ & Epidural analgesia $(\mathbf{n}=\mathbf{6 7 1})$ \\
\hline Median (IQR) gestational age at randomisation (weeks) & $37.8(35.5-39.2)$ & $37.1(35.3-39.0)$ \\
\hline Mean (SD) maternal age (years) & $31.5(5.1)$ & $31.7(4.8)$ \\
\hline White ethnic origin & $579(88)^{\star}$ & $561(90) \dagger$ \\
\hline Education $\geq$ higher & $281(52) \ddagger$ & $296(55) \S$ \\
\hline Median (IQR) BMI & $23.7(21.5-26.9) \boldsymbol{q}$ & $23.8(21.4-27.6)^{\star *}$ \\
\hline ASA classification: & $491(72)$ & \\
\hline 1 & $196(29)$ & $2101(69)$ \\
\hline 2 & & $329(49)$ \\
\hline Parity: & $323(47)$ & $342(51)$ \\
\hline 0 & $364(53)$ & $30(5)$ \\
\hline$\geq 1$ & $24(4)$ &
\end{tabular}

$\mathrm{IQR}=$ interquartile range; $\mathrm{BMI}=$ body mass index; $\mathrm{ASA}=\mathrm{American}$ Society of Anesthesiologists.

${ }^{*} 4.2 \%(29)$ missing.

†6.4\% (43) missing.

$\ddagger 21.7 \%$ (149) missing.

$\S 20.4 \%$ (137) missing.

\8.9\% (61) missing.

** $11.0 \%(74)$ missing. 
Table 2| Pregnant women allocated to patient controlled remifentanil or epidural analgesia in labour who received the other measured intervention

\begin{tabular}{lcc} 
& $\begin{array}{c}\text { Randomised to epidural analgesia, received } \\
\text { remifentanil }(\mathbf{n = 3 3})\end{array}$ & $\begin{array}{c}\text { Randomised to remifentanil, received epidural } \\
\text { analgesia }(\mathbf{n = 4 1 )}\end{array}$ \\
\hline Patient demand & 7 & 25 \\
\hline Physician assessment & 11 & 9 \\
\hline Contraindication for randomised treatment & $3^{*}$ & $3 \dagger$ \\
\hline Logistical problems & 9 & 1 \\
\hline Technical difficulties & 3 & 0 \\
\hline Unknown/missing & 0 & 3 \\
\hline
\end{tabular}

*Family history of Rendu-Osler-Weber syndrome, aortic valve stenosis, HELLP syndrome with thrombocyte count of 36 .

†Opioids administered $<6$ hours, initial maternal $\mathrm{SpO}_{2}<95 \%$, initial maternal temperature $>38^{\circ} \mathrm{C}$. 
Table 3| Area under curve for satisfaction with pain relief and pain scores during active labour and after start pain relief in pregnant women allocated to patient controlled remifentanil or epidural analgesia in labour

\begin{tabular}{|c|c|c|c|}
\hline \multirow[b]{2}{*}{ Measure (No of women per group) } & \multicolumn{3}{|c|}{ Mean area under curve } \\
\hline & Remifentanil & Epidural analgesia & Difference $(95 \% \mathrm{Cl})$ \\
\hline \multicolumn{4}{|l|}{ With missing AUC values imputed } \\
\hline Satisfaction with pain relief during active labour $(687 / 671)$ & 30.9 & 33.7 & $-2.8(-6.9$ to 1.3$)$ \\
\hline Satisfaction with pain relief after pain relief $\left(447 / 347^{\star}\right)$ & 25.6 & 36.1 & $-10.4(-13.9$ to -7.0$)$ \\
\hline Pain during active labour (687/671) & 30.9 & 27.2 & $3.8(0.92$ to 6.6$)$ \\
\hline Pain score after pain relief $\left(447 / 347^{\star}\right)$ & 26.7 & 20.3 & $6.4(3.5$ to 9.4$)$ \\
\hline \multicolumn{4}{|l|}{ Missing AUC values not imputed } \\
\hline Satisfaction with pain relief during active labour (394/290) & 27.2 & 37.6 & $-10.3(-14.6$ to -6.1$)$ \\
\hline Satisfaction with pain relief after pain relief $\left(316 / 198 \dagger^{\star}\right)$ & 25.5 & 41.3 & $-15.7(-20.2$ to -11.2$)$ \\
\hline Pain during active labour $(438 / 354)$ & 29.7 & 24.9 & $4.9(1.7$ to 8.1$)$ \\
\hline Pain score after pain relief $(345 / 220 \dagger)$ & 27.8 & 21.0 & 7.0 (3.3 to 10.7$)$ \\
\hline
\end{tabular}

*No who actually received pain relief.

†No who reported sufficient scores to calculate AUC and received pain relief. 
Table 4| Secondary outcomes for mean (SD) pain scores and scores for satisfaction with pain relief in pregnant women allocated to patient controlled remifentanil or epidural analgesia in labour. Missing values were not imputed

Remifentanil Epidural analgesia Difference $(95 \% \mathrm{Cl}) \quad \mathrm{P}$ value

\begin{tabular}{llccc} 
Satisfaction with pain relief during active labour & $5.1(2.3)$ & $5.9(2.5)$ & $-0.77(-1.1$ to -0.43$)$ & $<0.001$ \\
\hline Satisfaction with pain relief after pain relief & $5.3(2.3)$ & $7.0(2.5)$ & $-1.7(-2.1$ to -1.3$)$ & $<0.001$ \\
\hline Satisfaction with pain relief at request pain relief & 4.2 & 4.3 & $-0.12(-0.58$ to 0.35$)$ & 0.63 \\
\hline Highest satisfaction with pain relief after pain relief & $6.9(2.7)$ & $8.4(2.3)$ & $-1.5(-2.0$ to -1.1$)$ & $<0.001$ \\
\hline Pain during active labour & $6.0(1.9)$ & $5.2(2.3)$ & $0.74(0.46$ to 1.0$)$ & $<0.001$ \\
\hline Pain after pain relief & $6.1(1.9)$ & $4.2(2.3)$ & $1.9(1.5$ to 2.3$)$ & $<0.001$ \\
\hline Pain at request pain relief & $7.7(2.4)$ & $7.7(2.5)$ & $0.03(-0.32$ to 0.38$)$ & 0.87 \\
\hline Lowest pain score after pain relief & $4.0(2.6)$ & $1.7(2.3)$ & $2.3(1.9$ to 2.7$)$ & $<0.001$ \\
\hline
\end{tabular}


Table 5| Characteristics of labour in pregnant women allocated to patient controlled remifentanil or epidural analgesia according to intention to treat analysis. Figures are numbers (percentage) unless otherwise indicated

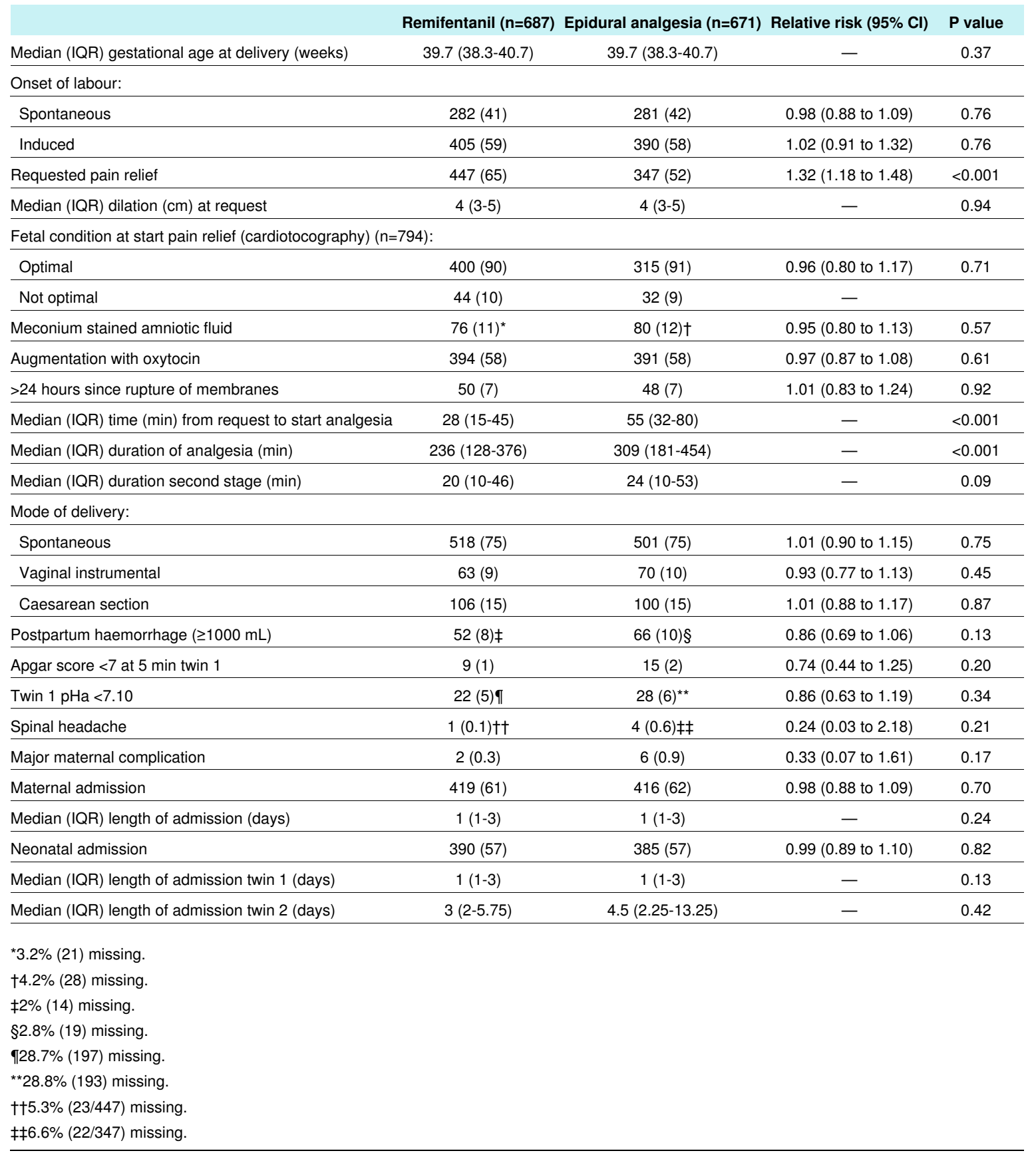


Table 6| Maternal side effects during administration of analgesia in pregnant women allocated to patient controlled remifentanil or epidural analgesia in labour. Figures are numbers (percentage) unless otherwise indicated

No (\%) with missing data

Remifentanil $(n=447)$ Epidural analgesia $(n=347)$ Relative risk $(95 \%$ Cl) P value Remifentanil Epidural

Temperature ${ }^{\circ} \mathrm{C}$ during labour:

\begin{tabular}{|c|c|c|c|c|c|c|}
\hline$>38^{\circ} \mathrm{C}$ & $35(9)$ & $55(18)$ & $0.66(0.50$ to 0.86$)$ & $<0.001$ & $41(9.2)$ & $35(10.1)$ \\
\hline \multicolumn{7}{|l|}{ Maximum reported: } \\
\hline Median (IQR) & $37.0(36.6-37.4)$ & $37.3(36.7-37.8)$ & - & $<0.001$ & $41(9.2)$ & $35(10.1)$ \\
\hline Range & $35.0-39.4$ & $35.1-40.4$ & - & - & - & - \\
\hline \multicolumn{7}{|l|}{ Saturation \%: } \\
\hline$<95 \%$ & $154(37)$ & $37(12)$ & 1.63 (1.46 to 1.82$)$ & $<0.001$ & $32(7.2)$ & $45(13.0)$ \\
\hline$<92 \%$ & $71(18)$ & $14(5)$ & $1.52(1.35$ to 1.71$)$ & $<0.001$ & $58(13)$ & $73(21)$ \\
\hline \multicolumn{7}{|l|}{ Minimum reported: } \\
\hline Median (IQR) & $95(93-97)$ & $97(96-98)$ & - & $<0.001$ & $58(13)$ & $73(21)$ \\
\hline Range & $50-100$ & $76-100$ & - & - & - & - \\
\hline Hypotension (<90 mm Hg systolic) & $29(7)$ & $38(12)$ & $0.75(0.57$ to 1.00$)$ & 0.03 & $26(5.8)$ & $19(5.5)$ \\
\hline Respiratory depression & $4(1)$ & $0(0)$ & - & 0.15 & $83(18.6)$ & $99(28.5)$ \\
\hline Nausea & $62(21)$ & $25(12)$ & 1.27 (1.09 to 1.49$)$ & 0.01 & $150(33.6)$ & $138(39.8)$ \\
\hline Vomiting & $55(18)$ & $28(13)$ & $1.16(0.97$ to 1.38$)$ & 0.12 & $145(32.4)$ & $134(38.6)$ \\
\hline Itching & $17(6)$ & $20(10)$ & 0.77 (0.54 to 1.10$)$ & 0.1 & $156(34.9)$ & $144(41.5)$ \\
\hline
\end{tabular}




\section{Figure}

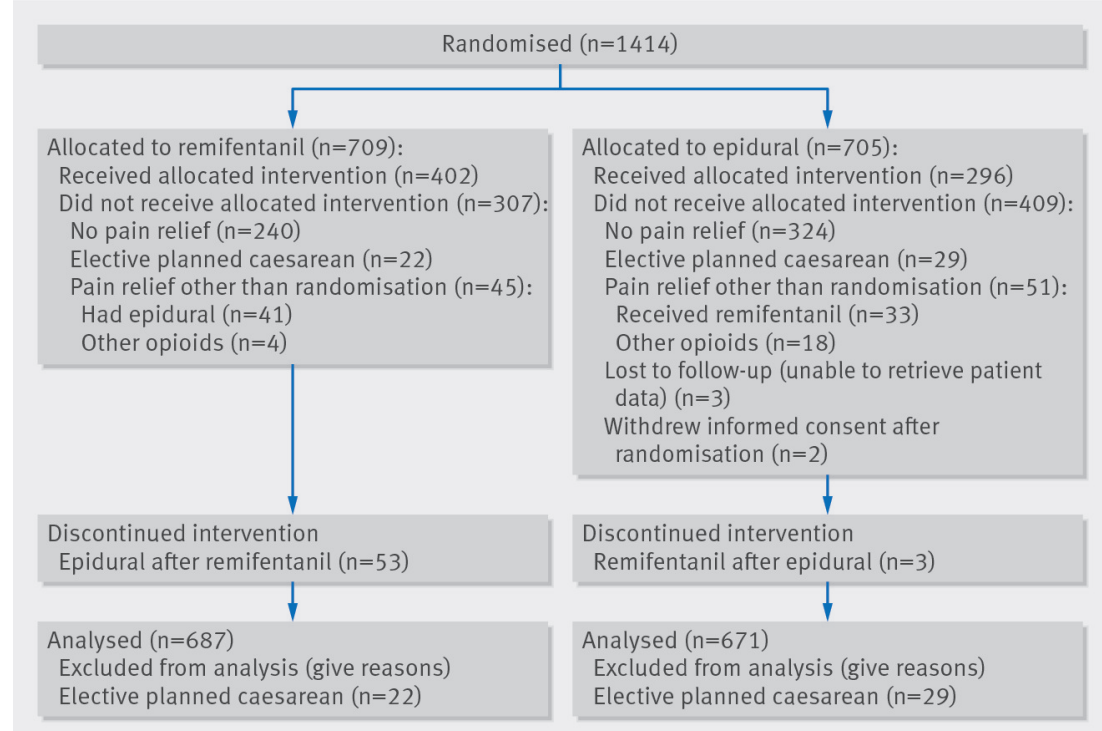

Randomisation and flow of pregnant women allocated to patient controlled remifentanil or epidural analgesia in labour 